\title{
Integrationsziel Politische Union: Überlegungen zu einem uneindeutigen Schlüsselbegriff
}

\author{
von Peter Graf Kielmansegg*
}

Politische Union, schon immer ein Schlüsselwort im Prozess der europäischen Einigung, rückte durch die Krise der Währungsunion erneut ins Zentrum der Diskussionen über die Zukunft Europas. Für zahlreiche Beobachter bildet sie sogar die Antwort auf die Krise. Aber ist ausreichend deutlich, was das ist - Politische Union? In der öffentlichen Debatte in Deutschland lassen sich zwei Argumentationslinien unterscheiden. Die eine, bescheidenere, zielt auf das, was nötig ist, damit die Währungsunion funktioniert. Die andere, ehrgeizigere, hat die Staatswerdung Europas im Visier. Das wird ungeachtet mancher Vagheit der Argumentation deutlich, wenn man fragt: Wer soll Träger der verfassunggebenden Gewalt sein? Welche Kompetenzen sollen auf Europa übergehen? Welche institutionelle Gestalt soll das vereinte Europa erhalten? Dem Ruf nach einer so verstandenen Politischen Union ist aber entgegenzuhalten: Ein Staat Europa ist in der vorhersehbaren Zukunft weder möglich noch wünschenswert. Es gilt, die Einzigartigkeit der Föderation europäischer Staaten ernst zu nehmen.

Political union has always been a keyword in the process of European integration. Recently, the crisis of the currency union has brought it back into the centre of discussions on the future of Europe. But do we know, what political union really means? In the German public debate two lines of reasoning can be distinguished. One, more modest, aims at making the currency union work. The other, more ambitious, aims at transforming the European Union into a state. This becomes clear, in spite of a good deal of vagueness, as soon as one asks: Who is to have the constitution-making power? Which responsibilities are to be transferred to Europe? How is the European Union to be shaped institutionally? However, the answer to the call for a political union understood in this way is: A state "Europe" is neither possible nor desirable in the foreseeable future. It is necessary to take the uniqueness of the federation of European states seriously.

\section{Einleitung}

Politische Union - wann immer in den sechzig Jahren europäischer Einigungsgeschichte darüber debattiert wurde, wohin das Einigungswerk führen solle, war dies ein Schlüsselwort. Schon 1953 stand es über einem Vertragsentwurf, der der

* Der folgende Essay nimmt nicht so sehr die wissenschaftliche als vielmehr die publizistische Debatte der vergangenen Jahre in den Blick. 
Montanunion und der Europäischen Verteidigungsgemeinschaft ein krönendes politisches Dach geben sollte. Regelmäßig kehrte es wieder. Und in der Gegenwart hat die Krise der Währungsunion es einmal mehr ganz ins Zentrum der politischen wie der intellektuellen Diskussionen katapultiert. Die Währungsunion, so das die Debatte beherrschende Standardargument, bedürfe der Politischen Union. Es sei der für die Krise verantwortliche Kardinalfehler der Gründung der Währungsunion gewesen, dass man ihr nicht die Politische Union zu- oder übergeordnet habe. Dieser Fehler müsse nun korrigiert werden. Daran, dass es natürlich auch ganz andere Lesarten der Krise gibt - etwa die, dass die Krise uns vor allem etwas über die derzeitigen Grenzen möglicher und sinnvoller Integration gelehrt habe -, sei nur erinnert. Uns geht es hier um die nachdrückliche Rückkehr der Chiffre Politische Union auf die Diskursagenda.

Wer nach der Politischen Union ruft, gibt zu verstehen, dass wir sie noch nicht haben. Was die Europäische Union in ihrer Gesamtheit angeht, so ist offensichtlich, dass es ein ziemlich absurder Sprachgebrauch wäre, sie als eine (derzeit noch) „unpolitische“ Union zu charakterisieren. Selbst ein oberflächlicher Blick auf ihr institutionelles Gefüge, ihre Zuständigkeiten, ihren Wirkungsraum, die Prozesse der Willensbildung in und zwischen ihren Organen zeigt: Die Europäische Union ist als Gesetzgeber in einem weiten Spektrum von Politikfeldern tätig und die Prozesse der Gesetzgebung laufen nach den Regeln der Politik ab - die EU ist ganz ohne Frage bereits eine politische Union. Allenfalls ließe sich von einem streng liberalen Standpunkt aus argumentieren, dass das Geschäft der Europäischen Union die Überwachung und Administration des Binnenmarktes sein sollte, die Verteidigung der vier Grundfreiheiten, die den Gemeinsamen Markt konstituieren - und dass Politik darüber hinaus von Übel sei. In dieser Auffassung begegnet uns ein „unpolitischer“ europäischer Binnenmarkt als ein liberales Ideal. Dass die Wirklichkeit damit angemessen beschrieben wäre, kann niemand, ob liberal gesinnt oder nicht, behaupten. Im Übrigen finden sich die, welche die Politische Union als zwingende Ergänzung der Währungsunion fordern, ja auch keineswegs im liberalen Lager, sondern eher auf der entgegengesetzten Seite des politischen Spektrums.

\section{Politische Union - ein Mittel zur Rettung der Währungsunion}

Was also ist gemeint, wenn nach der Politischen Union gerufen wird, obwohl die EU bereits eine politische Union ist? Dass der Begriff sich auf dem gegenwärtigen Integrationsniveau der Europäischen Union nicht mehr selbst erklärt, ist offenkundig. Es geht, das ist eine erste Antwort, offenbar um einige spezifische 
zusätzliche Kompetenzen für die EU, die als für die Funktionsfähigkeit der Währungsunion notwendig erachtet werden. Dass diese Kompetenzen das Etikett Politische Union erhalten, ist wenig hilfreich. Gesagt werden soll damit wohl, dass die Idee einer allein regelgesteuerten Währungsunion zu kurz greife; politische Mechanismen der Steuerung und Kontrolle müssten hinzutreten.

Wenig präzise Auskunft erhält man dann aber auf die Nachfrage, welche zusätzlichen Kompetenzen denn gemeint seien. Vielleicht liegt der Charme des Begriffs Politische Union ja eben darin, dass er Klarheit suggeriert und doch vieles offen lässt. Von der Notwendigkeit strikter gemeinschaftlicher Kontrolle über die nationalen Haushalte ist die Rede, auch von einer europäischen Wirtschaftsregierung. Das Stichwort Fiskalunion taucht auf, ebenso die Forderung nach einer europäischen Fiskal- und Wirtschaftspolitik. Vorsichtiger wird oft von einer verbindlichen Koordinierung der Wirtschafts- und Fiskalpolitiken der Mitgliedstaaten gesprochen. Was lässt sich aus diesen Stichworten herauslesen? Wenn denn die Annahme, die durch sie näher charakterisierte Politische Union werde die Währungsunion funktionsfähig machen, zu Ende gedacht ist, kann nur gemeint sein, es müsse europäische Interventions-, Steuerungs- und Kontrollrechte im Bereich der nationalen Haushalts- und Steuerangelegenheiten, auch der Wirtschaftspolitik geben, denen keine Vetorechte einzelner Mitgliedstaaten entgegenstehen.

Wie man sich das auf dem Feld der Wirtschaftspolitik vorzustellen hat, ist nicht leicht zu sehen. Schon die Frage, wie weit Regierungen Wirtschaftsentwicklungen steuern können und ob sie es anders als durch ordnungspolitische Vorgaben tun sollten, wird ja von Land zu Land - da spielen kulturelle Prägungen eine wichtige Rolle - und von Parteilager zu Parteilager durchaus unterschiedlich beantwortet. Was bedeutet das für eine wirksame Koordinierung? Sind europäische Weisungsbefugnisse impliziert? Sollen europäische Instanzen den Mitgliedstaaten Konjunkturprogramme vorschreiben können? Sollen sie anordnen können, dass die Mitgliedstaaten sich an ganz bestimmten ordnungspolitischen Mustern orientieren, etwa in der Ausgestaltung der Arbeitsbeziehungen, die ja Schlüsselbedeutung für das Wirtschaftsleben eines Landes haben? Sollen sie verbindliche industriepolitische Vorgaben machen dürfen? Gleichgültig, ob man von einer europäischen Wirtschaftsregierung oder von der verbindlichen Koordinierung der Wirtschaftspolitiken der Mitgliedstaaten spricht, es ist schwierig, hinter diesen Vokabeln ein in sich schlüssiges, praktikables und dazu noch einigermaßen marktkonformes Programm europäischen politischen Handelns ausfindig zu machen. 
Etwas leichter lässt sich Klarheit beim Stichwort Fiskalpolitik gewinnen. Die zurückhaltendste Form einer europäischen Kontrolle der Haushalte der Mitgliedstaaten wäre es, das Volumen der mitgliedstaatlichen Gesamthaushalte zu überwachen, um sicherzustellen, dass die Mitgliedsländer sich nicht übermäßig verschulden. Eine entsprechende Verpflichtung hat bekanntlich von Anfang an zum Konzept der Währungsunion gehört. Sie hat nicht viel bewirkt. Neu wäre die Fähigkeit zu einer wirksamen europäischen Intervention. Wirksam heißt: nicht von den Betroffenen und - wichtiger noch - potentiell Betroffenen blockierbar. Für direkte Interventionen in das Budget, Anordnungen zu Umschichtungen, Streichungen und was sich sonst denken lässt, gilt allerdings: Sie greifen in den Kern der Verantwortung der mitgliedstaatlichen Regierungen und Parlamente für das eigene Land ein. Mit guten Gründen hat das Bundesverfassungsgericht in seinen Urteilen zum Fortgang der europäischen Integration seit dem Maastrichter Vertrag inzwischen mehrfach darauf hingewiesen, dass das Budgetrecht das entscheidende Recht eines jeden Parlamentes sei und dass dieses Recht jedenfalls nach dem Willen des Grundgesetzes nicht substanzlos werden dürfe. Unabhängig von allen verfassungsrechtlichen Vorgaben, die von Mitgliedsland zu Mitgliedsland unterschiedlich aussehen, wird man sagen müssen: Ein Weisungsrecht der EU, das sich auf die Inhalte der mitgliedstaatlichen Budgets erstreckt, würde die Mitgliedstaaten in der innersten Zone ihres Selbstregierungsrechtes partiell entmündigen. Was indirekte Einwirkungen in Gestalt von Sanktionen angeht, so ist zu bedenken: Strafzahlungen müssen eine abschreckende Höhe erreichen, um wirksam zu sein, hätten dann aber in dem betroffenen Land Wirkungen, die die EU nicht wünschen, vielleicht nicht einmal verantworten kann.

Man sieht: Mit der Forderung nach Durchsetzung von fiskalischer Disziplin in den Mitgliedstaaten lassen sich präzisere Vorstellungen verbinden als mit dem Stichwort Wirtschaftsregierung. Aber ob diese Forderung einen gangbaren, vernünftigen Weg der Fortentwicklung der Europäischen Union weist, ist eine andere Frage. Die zurückhaltendere Rede von einer verbindlichen Koordination der Fiskalpolitik bleibt die Antwort auf die Frage schuldig, wer die verbindlichen Entscheidungen trifft und wie viel Angleichung der Begriff Koordination meint. Erinnert man sich daran, dass mit der verbindlichen Koordination der Fiskalpolitik ja die nach allgemeiner Überzeugung noch fehlenden Voraussetzungen für die Funktionsfähigkeit der Währungsunion geschaffen werden sollen, so wird deutlich, dass auch die verbindliche Koordination sehr rasch in jene Problemzonen hineinführen kann und muss, in die das zuvor erörterte Disziplinierungsinstrumentarium führt. 
Dass das strategische Konzept, mit zusätzlichen europäischen Kompetenzen der Einwirkung auf die Budgets der Mitgliedstaaten der Währungsunion auf die Beine zu helfen, vom ersten Schritt gedanklicher Konkretisierung an auf Schwierigkeiten stößt, liegt in ihm selbst begründet. Aber in diesen Schwierigkeiten spiegelt sich auch wider, dass es sehr zweifelhaft ist, ob es denn überhaupt das Fehlen bestimmter Kontroll- und Interventionskompetenzen war, das die Währungsunion in ihre Krise geführt hat. Anders formuliert: ob diese Kompetenzen das Fehlen ganz anderer, viel elementarerer Voraussetzungen der Funktionsfähigkeit der Währungsunion kompensieren können.

\section{Politische Union - ein Programm zur Staatswerdung Europas}

Man mag die bisher skizzierte, sehr stark auf die dem Projekt Währungsunion innewohnende Logik fixierte Argumentationslinie, die im Wesentlichen auf eine Stärkung der Kommission hinausläuft, als bürokratisch oder technokratisch charakterisieren, mithin die Politische Union, die diese Argumentationslinie im Visier hat, als eher bürokratisch-technokratische Variante einer Politischen Union sehen. Das geschieht auch immer wieder in durchaus kritischer Absicht. So ist es nicht überraschend, dass es den Ruf nach der Politischen Union im Gefolge der Krise auch noch in einer ganz anderen Stimmung, Färbung und Ausrichtung gibt. Der Ausgangspunkt bleibt dabei: die Krise der Währungsunion. Aber es geht in dieser zweiten Argumentationslinie nicht eigentlich um Reparatur, darum, die Währungsunion nachträglich auf ein immer noch schmales, aber doch tragfähiges Fundament zu setzen. Es geht um die Krise der Währungsunion als Chance - als Chance nämlich, einen großen Integrationsschritt nach vorn zu tun, der, Währungsunion hin, Währungsunion her, ohnehin längst fällig sei, um das Projekt Vereintes Europa seinem Ziel ein entscheidendes Stück näher zu bringen.

Die Krise wird hier als Katalysator in den Dienst des europäischen Projektes gestellt. In ihr ist gewissermaßen die List der historischen Vernunft am Werk. Sie lässt die Europäer im Nachhinein wahrnehmen, dass ein Integrationsschritt, den sie getan haben, weitere Integrationsschritte entweder zwingend erforderlich macht oder doch nahe legt, an die sie beim ersten Schritt nicht, noch nicht gedacht haben; Integrationsschritte, die nun aber nicht nur nachliefern, was der Währungsunion in ihrer ersten unvollkommenen Gestalt noch fehlte, sondern auf eine neue Stufe europäischer Gemeinsamkeit hinaufführen. Die List der historischen Vernunft, so diese Sicht der Krise, kann dem europäischen Projekt über die Zögerlichkeiten der europäischen Völker hinweghelfen, die es gefährden.

Mit zwei Schlüsselwörtern wird jenes fortgeschrittene Europa, das ein von der 
Krise der Währungsunion ausgelöster Integrationsschub hervorbringen kann und soll, gekennzeichnet: Demokratie und Solidarität. Die neuen Kompetenzen, die der Europäischen Union vor allem auf dem Felde der Wirtschafts-, Fiskal- und Sozialpolitik zuwachsen müssten, seien, so wird argumentiert, einzubetten in eine umfassende Demokratisierung der Union; sie müssten zudem verbunden sein mit einer umfassenden Institutionalisierung europäischer Solidarität. Was mit dem Ruf nach europäischer Demokratie und europäischer Solidarität im Einzelnen gemeint ist, bleibt zunächst einmal offen. Wie würde das Institutionen- und Regelgefüge einer umfassend demokratisierten Europäischen Union aussehen? Welche Zuständigkeiten müssten auf die Europäische Union übertragen werden, damit sie zur Solidargemeinschaft wird? Wie weit sollte die umfassende Solidarität reichen? Wie immer die genaueren Antworten lauten, es handelt sich - von dieser These gehen die folgenden Überlegungen aus - der Sache nach und zu Ende gedacht um ein Plädoyer für die Staatswerdung Europas. Politische Union - das ist in diesem Argumentationskontext als Chiffre für ein Programm zu lesen, das auf einen Staat Europa abzielt. ${ }^{1}$

Bemerkenswerterweise spricht das gegenwärtig niemand klar aus. Im Gegenteil. Selbst dort, wo das Ziel, die europäische Integration möglichst rasch bis zur Staatswerdung Europas vorwärts zu treiben, ganz deutlich hervortritt, fehlt der vorsorgliche Hinweis nicht, an einen Staat Europa sei nicht gedacht. ${ }^{2}$ Warum diese eigentümliche Scheu, das eigentlich Gemeinte auch auszusprechen? Taktische Gründe mögen im Spiel sein. Dass das politische Programm, aus der Europäischen Union einen Staat zu machen, heute und auf absehbare Zeit in Europa nicht mehrheitsfähig ist, weder unter den Staaten noch unter den Völkern, ist ja offenkundig. Da mag es als klug erscheinen, für dieses Ziel zu werben, ohne das Kind beim Namen zu nennen. Wichtiger aber ist wohl, dass die Protagonisten einer Politischen Union in dieser anspruchsvollen Version sich ihrer hochgesteckten Ziele ungeachtet als Realisten sehen, das aus ihrer Sicht Sinnvolle als möglich begreifen möchten. Vielleicht denken sie auch die Implikationen ihres Programms nicht wirklich zu Ende, täuschen sich also selbst, weil auch sie sich nicht ganz sicher sind, ob sie den Staat Europa wirklich wollen sollen.

1 Gelegentlich greift der Ruf nach einem Integrationssprung auch wieder auf das alt-ehrwürdige Etikett „Vereinigte Staaten von Europa“ zurück (Reding, V.: Der neue Bund, in: Frankfurter Allgemeine Zeitung, 25.02.2013, 7). Ob man es will oder nicht, auch diese Begrifflichkeit impliziert die Staatlichkeit Europas. Man kann nicht von „Vereinigten Staaten“ sprechen, ohne auf die Vereinigten Staaten von Amerika zu verweisen. Die USA aber sind ein Bundesstaat.

2 So in dem Beitrag Bofinger, P./Habermas, J./Nida-Rümelin, J.: Einspruch gegen die Fassadendemokratie, in: Frankfurter Allgemeine Zeitung, 04.08.2013, 33. 
Wenn es sich so verhält; wenn die Chiffre Politische Union für das Ziel steht, die Integration möglichst rasch bis zur Staatswerdung Europas weiterzutreiben, dieses Ziel aber in der voraussehbaren Zukunft politisch keine Chancen hat, lohnt es sich dann überhaupt, mit der Entschlüsselung dieser Chiffre fortzufahren? Die Antwort lautet kurz und bündig: Ja! Auch wenn der Staat Europa gegenwärtig auf keiner politischen Agenda steht, die intellektuellen Diskurse müssen alle denkbaren Optionen, die Europa hat, in den Blick nehmen. Sie müssen sie ausbuchstabieren und müssen ihre Wünschbarkeit ebenso erörtern wie ihre Möglichkeit. Dies aus mindestens zwei Gründen. Zum einen sind die intellektuellen europapolitischen Diskurse von heute mitverantwortlich für die politische Agenda von morgen. Zum andern kann zwischen den politisch aktuellen Nahzielen und den denkbaren Fernzielen des Integrationsprozesses gar keine scharfe Grenzlinie gezogen werden. Jeder nächste Integrationsschritt stellt eine Weiche hin auf eine mögliche oder eine Weiche weg von einer möglichen Integrationszukunft. Im Naheliegenden ist also immer auch das Fernliegende mitzubedenken.

An diesem Schluss ändert die Tatsache nichts, dass der EU gerade die gemeinhin als konstitutiv angesehenen Attribute der Staatlichkeit auch nach 60 Jahren Integrationsgeschichte allesamt noch fehlen: Sie verfügt über keine eigenen Gewaltmittel, sie erhebt keine Steuern, sie entscheidet nicht autonom über ihre Zuständigkeiten. Aber die Idee der Staatswerdung hat das europäische Projekt von seinen Anfängen an beleitet. Mag sie auch im Lauf der Jahrzehnte an Strahlkraft und vor allem - mit jedem neuen Erweiterungsschub - an Plausibilität verloren haben, sie wird es weiter begleiten. Das Nachdenken über die Zukunft Europas wird sich nicht einfach von ihr verabschieden. Und es könnte durchaus sein, dass diese Idee im kontinuierlichen, wenig merklichen, unumkehrbaren Fortschreiten des Integrationsprozesses, in der Fortentwicklung der Föderation gewissermaßen von selbst wieder politische Aktualität gewinnt, ohne explizit von irgendjemandem auf die politische Agenda gesetzt worden zu sein. Die Krise der Währungsunion hat gezeigt, wie das geschehen kann.

Es bleibt also dabei: Staatswerdung Europas, das Thema, mit dem uns die Rückkehr des Topos von der Politischen Union ins Zentrum der Europadiskussion letztlich konfrontiert, ist ein auch aktuelles Thema. Es aufzunehmen heißt zunächst einmal, nach genaueren Konturen der Idee der Staatswerdung Europas zu fragen. Ob es eine vernünftige Idee ist, wird anschließend zu erörtern sein. 


\section{Die verfassunggebende Gewalt}

Der Staat ist definiert durch das Letztentscheidungsrecht und die Letztentscheidungsmacht in bestimmten territorialen Grenzen, auch wenn die Letztentscheidungsmacht natürlich immer faktischen Restriktionen unterliegt. Das Letztentscheidungsrecht ist an erster Stelle das Recht, sich eine Verfassung zu geben. Die Frage nach dem Träger der verfassunggebenden Gewalt, ist mithin die erste, die gestellt werden muss, um den Charakter einer Politischen Union Europa zu bestimmen. Die derzeitige Rechtslage ist eindeutig. Die Mitgliedstaaten, so lautet die bildhafte Formel, sind die Herren der Verträge, und die Verträge bilden die Verfassung der Europäischen Union. Änderungen der Verträge werden von den Regierungen der Mitgliedstaaten ausgehandelt und müssen von den Parlamenten der Mitgliedstaaten ratifiziert werden. Dabei gilt der Grundsatz der Einstimmigkeit. In Bundesstaaten hingegen - und der Staat Europa ist natürlich als Bundesstaat gedacht - liegt die verfassungsändernde Gewalt beim Bundesstaat. Die Gliedstaaten wirken nach unterschiedlichen Regeln mit, tun dies aber eingebunden in ein Regelwerk, nach dem Mehrheiten entscheiden, Mehrheiten in den gesetzgebenden Organen des Bundes, Mehrheiten gegebenenfalls des Bundesvolkes, Mehrheiten der mitwirkenden Länder.

Selbst in den Vereinigten Staaten von Amerika, in denen die Gliedstaaten eine besonders starke Stellung im Verfahren der Verfassungsänderung haben, ist das nicht anders (Art. 5 der Verfassung). Drei Viertel aller Gliedstaaten, von den derzeit 50 also 39, müssen einer vom Kongress mit Zweidrittelmehrheit vorgeschlagenen Verfassungsänderung zustimmen, damit sie in Kraft treten kann. Das ist eine sehr hohe Hürde, aber die Entscheidung bleibt eine Mehrheitsentscheidung. Einzig für die gleiche Vertretung aller Staaten, der kleinen wie der großen, im Senat gilt, dass dieser Grundsatz nur mit der Zustimmung aller Staaten aufgehoben werden kann. Die Herkunft der Vereinigten Staaten aus einer staatenbündischen Föderation ist hier (wie an einigen anderen Stellen in der Verfassung) noch sehr greifbar. Wenn man den letzten Satz des Art. 5 liest, ahnt man, dass eben dieser Satz entscheidend für das Gelingen der Verwandlung eines Staatenbundes in einen Bundesstaat war. Gleichwohl: Die verfassungsändernde Gewalt wird auch in diesem Punkt nicht einfach den Gliedstaaten überlassen. Es wird nur jedem einzelnen Gliedstaat in dieser (wie man irrtümlich meinte) Existenzfrage ein Vetorecht zugestanden.

Die tatsächlichen Verhältnisse freilich sind nicht so einfach wie die Rechtslage. Faktisch nimmt die Europäische Gemeinschaft erheblichen Einfluss auf die Fortentwicklung der europäischen Verfassung. Zwei europäische Institutionen, die 
Kommission und der EuGH, kommen hier ins Spiel. Die Kommission besitzt das Initiativrecht für die europäische Gesetzgebung, genauer: die Verträge geben ihr ein Initiativmonopol. Das allein schon verleiht ihr ein starkes Gewicht in den politischen Prozessen, in denen die Reichweite europäischer Zuständigkeitsnormen faktisch bestimmt wird. Dass die Zuständigkeiten der EU letztlich über die Ziele des Integrationsprozesses definiert sind, erweitert den Handlungsspielraum der Kommission noch einmal zusätzlich. Sie nutzt diese Möglichkeiten natürlich, ihrem Eigeninteresse folgend, um den Raum ihrer Zuständigkeiten stetig auszuweiten; an dem Gebäude europäischen Rechts stetig weiterzubauen.

Der EuGH legt das europäische Primärrecht, so wie es in den Verträgen niedergelegt ist, die Europäische Verfassung also, abschließend verbindlich aus. Das heißt, er befindet darüber, ob europäisches Sekundärrecht oder mitgliedstaatliches Recht mit den Verträgen vereinbar ist. Und dabei entscheidet er im Besonderen im Wege der Auslegung auch über die Reichweite der Normen, die der Gemeinschaft Kompetenzen zuweisen. Das ist, streng genommen, noch keine Kompetenz-Kompetenz. Denn die Verfassungsnormen über die Verteilung der Zuständigkeiten sind dem Gericht in Gestalt der Verträge ja vorgegeben. Aber davon war eben schon mit Blick auf die Kommission die Rede - angesichts des dynamischen, auf Ziele ausgerichteten, auf Entwicklung angelegten Charakters der europäischen Föderation und ihrer Verfassung verbirgt sich in der Auslegungsmacht des EuGH tatsächlich ein gewichtiges Stück KompetenzKompetenz. Anders formuliert: In der Institution des Europäischen Gerichtshofes, in gewissem Sinne sogar schon in der der Kommission, hat die Föderation bereits jetzt Anteil an der verfassunggebenden Gewalt. Und der EuGH macht von seiner Auslegungsmacht durchaus auch in diesem Sinne Gebrauch. Indem er die Verträge auslegt, schreibt er sie faktisch weiter. Die Mitgliedstaaten sind nur in einem eingeschränkten Sinn Herren der Verträge.

Das Bundesverfassungsgericht hat dem vom EuGH für sich in Anspruch genommenen Auslegungsprimat bekanntlich die Auffassung entgegengesetzt, die Übertragung von Hoheitsrechten auf die EU sei ein Akt mitgliedstaatlicher (Verfassungs-)Gesetzgebung. ${ }^{3}$ Die Frage, welche Rechte ein Mitgliedstaat tatsächlich übertragen habe und seiner Verfassung gemäß habe übertragen dürfen, müsse deshalb im Rahmen der Verfassungsordnung des Mitgliedstaates geprüft und letztlich auch entschieden werden, durch Auslegung des Übertragungsgesetzes

3 So vor allem das Urteil zum Maastricht-Vertrag 1993 (BVerfGE 89, 155ff.) und das Urteil zum Lissabon-Vertrag 2009 (BVerfGE 123, 267ff.). 
und der ihm zugrunde liegenden Verfassungsbestimmungen. Beide Argumentationen sind logisch schlüssig - die des EuGH, der geltend machen kann, dass nur eine abschließend verbindliche europäische Kompetenz der Auslegung des europäischen Rechtes einen einheitlichen europäischen Rechtsraum schaffen könne, und die des Bundesverfassungsgerichts, das sich darauf berufen kann, dass die Mitgliedstaaten die Beantwortung der Frage, welche Kompetenzen sie auf die EU übertragen haben (von dem Problem der Verfassungsmäßigkeit dieser Übertragung zu schweigen), nicht einfach einer dritten Instanz überlassen können, wenn nicht der Grundsatz, nach dem alle Kompetenzen der EU sich ausschließlich aus expliziter Übertragung von den Mitgliedstaaten herleiten, faktisch in sein Gegenteil verkehrt werden soll. Dass hier Logik auf Logik trifft, theoretisch unversöhnlich, zeigt, wie spannungsreich die in ihrer Struktur einzigartige europäische Föderation ist.

Und wie gehen die Plädoyers für die Politische Union mit dem Thema verfassunggebende bzw. verfassungsändernde Gewalt um? Es ist auffallend, wie selten sie dieses Thema auch nur berühren, etwa den eben beschriebenen eigentümlichen Zustand einer faktisch bereits (wenn auch ungleichgewichtig) geteilten verfassungsändernden Gewalt in den Blick nehmen. Aber gelegentlich gibt es doch Äußerungen, die erkennen lassen, dass es bei diesem Zustand nicht bleiben kann und soll, wenn das Projekt Politische Union bis zur Staatswerdung Europas weitergedacht wird. Oder richtiger umgekehrt, dass zum Projekt der Politischen Union auch der volle Übergang der verfassungsändernden Gewalt auf die Föderation gehört, womit unzweideutig klargestellt ist, dass dieses Projekt auf die Staatswerdung Europas angelegt ist. In einem Diskussionsbeitrag von Wolfgang Schäuble etwa kann man nachlesen: „Klare, effiziente Kompetenzabgrenzung setzt allerdings die Bereitschaft der Mitgliedstaaten voraus, Teile von Souveränität irreversibel an die europäische Ebene abzugeben. Das Diktum, das die Mitgliedstaaten Herren der Verträge bleiben, ist damit auf die Dauer nicht zu vereinbaren.“4 Die Logik des Schlusses mag zweifelhaft sein, die Aussage ist eindeutig.

In einem anderen Diskussionsbeitrag heißt es, im Zuge der Übertragung weiterer Hoheitsrechte auf die EU werde ihre konsequente Parlamentarisierung notwendig, ,bis zu dem Punkt, an dem die Souveränitätsfrage sich zugunsten der Europäischen Union klärt“. ${ }^{5}$ Die Souveränitätsfrage - das ist für den Binnenbereich

4 Schäuble, W.: Institutioneller Wandel und europäische Einigung, in: Frankfurter Allgemeine Zeitung, 21.01.2013, 14.

5 Winkler, H.A.: Vom Staatenverbund zur Föderation, in: Frankfurter Allgemeine Zeitung, 13.06.2012, 6. 
im Kern die Frage nach der verfassungsändernden Gewalt. Ein drittes Beispiel: Ein Plädoyer für einen neuen europäischen Verfassungskonvent mündet in den Vorschlag ein, die Entscheidung über den Konventsentwurf von einer zweifachen Mehrheit treffen zu lassen, einer Mehrheit der Unionsbürger und einer Mehrheit der Mitgliedstaaten. ${ }^{6}$ Ein europäisches Verfassungsplebiszit also, ergänzt durch einen Mehrheitsentscheid der Staaten. Das ist ein Entscheidungsmodus, der die verfassunggebende Gewalt eindeutig in die Hände der Föderation legt, wo sie nach der Logik dieser Weichenstellung als verfassungsändernde Gewalt dann auch verbleibt. Daran, dass ein solcher Entscheidungsmodus ein entscheidender Schritt, ja der entscheidende Schritt auf dem Weg zur Staatswerdung Europas wäre, kann kein Zweifel bestehen. Wer die verfassunggebende und die verfassungsändernde Gewalt innehat, verfügt über die KompetenzKompetenz, mithin die Zuständigkeit, über die Verteilung der Kompetenzen zu entscheiden. Und wer darüber entscheidet, ist in einer bündischen Ordnung der Souverän.

\section{Die Kompetenzen}

Mögen sich die meisten Plädoyers für eine Politische Union, was den Träger der verfassunggebenden Gewalt angeht, auch nur undeutlich äußern, mehr Zuständigkeiten für die EU fordern sie alle ein. Tatsächlich ist die Forderung, die EU müsse mehr Kompetenzen erhalten, in der Regel ihr Kern. Die Frage, welche es denn sein sollen, wird freilich nur sehr unbestimmt beantwortet. Diese Unbestimmtheiten müssen wir genauer betrachten. Aber zunächst ist eine Vorfrage zu klären. Kommt es bei der EU auf Zuständigkeitsregelungen überhaupt wesentlich an? Der Zweifel daran mag überraschen - sind Zuständigkeitsregelungen nicht konstitutiv für alle Föderationen? Aber er ist keineswegs aus der Luft gegriffen.

Die Vertragsverfassung der EU unterscheidet zwischen Zielen (Art. 3 EUV) und Zuständigkeiten (Art. 4 EUV) der Gemeinschaft, eine Unterscheidung, die andere föderale Verfassungen so nicht kennen. Die Ziele sind weit und umfassend formuliert. Friede, Wohlergehen der Völker, ausgewogenes Wirtschaftswachstum, Vollbeschäftigung, sozialer Fortschritt, Gleichstellung von Mann und Frau, Schutz der Rechte des Kindes, Solidarität zwischen den Mitgliedstaaten - das ist nur eine Auswahl aus dem Strauß hehrer Vorgaben, auf die Art. 3 EUV die Union verpflichtet. Zwar heißt es im letzten Absatz, die Union verfolge ihre Ziele

6 Landfried, C.: Wir wollen mehr Hannah Arendt wagen, in: Frankfurter Allgemeine Zeitung, 04.02.2014, 30 . 
entsprechend den Zuständigkeiten, die ihr in den Verträgen übertragen seien. Aber Art. 352 AEUV stellt unzweideutig klar, dass die Ziele Vorrang vor den Zuständigkeiten haben: „Erscheint ein Tätigwerden der Union im Rahmen der in den Verträgen festgelegten Politikbereiche erforderlich, um eines der Ziele der Verträge zu verwirklichen, und sind in den Verträgen die hierfür erforderlichen Befugnisse nicht vorgesehen, so erlässt der Rat einstimmig auf Vorschlag der Kommission und nach Zustimmung des Europäischen Parlamentes die geeigneten Vorschriften“ (Art. 352 Abs. 1 Satz 1 AEUV). Gewiss, jeder Staat hat hier ein Vetorecht. Aber Konstellationen, in denen sich kein Staat - die Interessenlage von Kommission und Parlament ist ohnehin klar - einer ad-hoc-Erweiterung der Unionskompetenzen explizit entgegenstellen mag, sind auf bestimmten Politikfeldern keineswegs unwahrscheinlich.

Zudem ist hier an bereits Gesagtes noch einmal zu erinnern. Allein das Initiativrecht der Kommission und das Auslegungsmonopol des EuGH bewirken schon, dass die Zuständigkeiten der Union kontinuierlich in Richtung auf die Ziele ausgeweitet werden - ganz unabhängig von der Kompetenzerweiterungsklausel des Art. 352 AEUV. Zwar spiegelt sich in dem Katalog von Zuständigkeiten des Art. 4 EUV deutlich wider, dass die EU im Kern eine Wirtschaftsgemeinschaft ist. Aber es gibt in der Welt von heute kaum einen Lebensbereich, in den die Wirtschaft nicht hineinwirkt; oder umgekehrt: von dem nicht Wirkungen auf das Wirtschaftsleben ausgehen. Deswegen ist eine umfassende Kompetenz zur Gesetzgebung in wirtschaftlichen Angelegenheiten so etwas wie eine Brücke zu einer Art von Beinahe-Allzuständigkeit. Zumindest kann sie so verstanden und genutzt werden.

Haben wir es in der EU also überhaupt mit einer einigermaßen stabilen Kompetenzverteilung zu tun oder nicht viel eher mit einer stetigen Dynamik der Erweiterung der Handlungsspielräume der EU ohne Änderung der vertraglichen Grundlagen? Das ist eine Vermutung, die Einiges für sich hat. Sie muss freilich in Relation gesetzt werden zu der Feststellung, dass diese Dynamik dort, wo die Staaten ein starkes, anhaltendes und gemeinsames Interesse daran haben, Zuständigkeiten nicht aus der Hand zu geben, an Grenzen stößt, die sie nicht überwinden kann. Die Außen- und Sicherheitspolitik ist eine solche Domäne der Mitgliedstaaten, die Steuerhoheit eine andere. Zuständigkeitsregelungen, heißt das, sind bei aller Eigenart dieses besonderen föderalen Gebildes keineswegs bedeutungslos. Und so hat auch die Frage, was der Ruf nach einer Politischen Union für die Zuständigkeitsverteilung bedeute, ihren Sinn.

Im Allgemeinen fällt die Begründung der Forderung nach einer Ausweitung der EU-Kompetenzen knapp und pauschal aus: Der Nationalstaat sei zu klein gewor- 
den für die Herausforderungen des 21. Jahrhunderts. Für welche Herausforderungen das tatsächlich gilt und in welchen Bereichen die Problemlösungsfähigkeit traditioneller, kleinerer politischer Einheiten der von kontinentalen, sehr inhomogenen Großgebilden eher überlegen ist, wird selten differenziert erörtert. Machte man sich die Mühe, den gewaltigen acquis communitaire genau durchzumustern, so würde man vieles finden, was zur Fähigkeit Europas und seiner Staaten, sich in einer globalisierten Welt zu behaupten, überhaupt nichts beiträgt - die Antidiskriminierungsrichtlinie etwa, um nur dieses eine prominente Beispiel zu nennen.

Der aktuelle Ruf nach der Politischen Union hat allerdings auch in dem, was er zur Zuständigkeitsverteilung sagt, einen sehr viel spezifischeren Anknüpfungspunkt, eben die Krise der Währungsunion. Die immer wiederkehrenden Stichworte sind uns bereits vertraut: europäische Wirtschaftsregierung, Wirtschaftsund Fiskalunion, zumindest verbindliche Koordination der Wirtschafts- und Fiskalpolitik oder spezifischer der Haushaltspolitik. Auch von den Schwierigkeiten, diese Stichworte in ihrer Unbestimmtheit in konkrete Unionszuständigkeiten zu übersetzen, war schon die Rede. Ob sich in ihnen ein Programm der Staatswerdung Europas verbirgt, hängt aber von eben dieser Übersetzung ab. Klar ist nur: Wenn die Rede von der Fiskalunion oder von der europäischen Fiskalpolitik auch ein Recht der Union, Steuern zu erheben, meint, dann hat sie tatsächlich die Staatswerdung Europas im Visier.

Ähnliches gilt für die Forderung nach einer europäischen Sozialunion oder einer europäischen Sozialpolitik, die in den anspruchsvolleren Bestimmungen dessen, was Politische Union bedeute, immer wieder auftaucht. Zielt diese Forderung nur auf verbindliche europäische Mindeststandards, so ist allenfalls anzumerken, dass für Mindeststandards, jedenfalls im europäischen Raum, vermutlich schon der demokratische politische Prozess in den Mitgliedstaaten selbst sorgt. Zielt sie darüber hinaus, so dringt sie in Kernzonen der Eigenstaatlichkeit der Mitgliedstaaten ein. Angesichts großer, historisch wie kulturell begründeter Unterschiede zwischen den Systemen sozialer Sicherung der Staaten Europas wäre der Versuch einer über die Setzung von Mindeststandards hinausgehenden Vereinheitlichung ein ebenso unnötiges wie unsinniges Unternehmen. Sinnvolle Angleichungen können und sollten sich durch wechselseitiges Lernen vollziehen.

Wer von einer europäischen Sozialunion spricht, wird also vor allem sagen wollen, dass die Finanzierung der Systeme sozialer Sicherung in den Mitgliedstaaten zumindest partiell europäisiert werden sollte. Das würde Umverteilung zwischen den Mitgliedstaaten weit über das hinaus, was gegenwärtig etwa über die Strukturfonds umverteilt wird, bedeuten; Umverteilung, bei der es nicht nur um Steu- 
ermittel, sondern auch um Beiträge gehen würde, für die sich ersichtlich andere Fragen stellen. Die Umverteilung von Mitteln, die durch die Union für die soziale Sicherung aufgebracht werden, müsste, wenn es sich nicht nur um marginale Summen handelt, notwendigerweise mit dem Recht der Union einhergehen, auf die Regelwerke der Erhebung und Verteilung dieser Mittel einzuwirken. Denn wenn die Systeme sozialer Sicherung partiell über die Union finanziert werden, kann es nicht mehr Sache der Mitgliedstaaten allein sein, zu entscheiden, nach welchen Regeln diese Systeme arbeiten. Das aber bedeutet: Auch die Forderung, die europäische Föderation im Rahmen des Aufbaus einer Politischen Union zur Sozialunion auszugestalten, impliziert die Staatswerdung Europas. Und dies umso deutlicher, als sie ja stillschweigend eine Art von Solidaritätsbereitschaft gesamteuropäisch voraussetzt, wie wir sie bisher nur aus den Nationalstaaten, und nicht einmal dort immer verlässlich, kennen. Ohne ein zur unreflektierten Selbstverständlichkeit gewordenes gemeinsames Verständnis der Reichweite von Solidarität in einer Bürgergemeinschaft gibt es keine tragfähige Akzeptanz von verordneter Umverteilung, auf die Demokratien angewiesen sind.

Alles in allem: Genauen Aufschluss darüber, auf welche Erweiterungen von Unionszuständigkeiten der Ruf nach einer Politischen Union zielt, gibt die öffentliche Debatte nicht. Sie begnügt sich mit Richtungshinweisen. Aber denkt man diese Hinweise weiter, so wird sehr rasch klar, dass sie auf mehr als einem Politikfeld die Staatswerdung Europas im Visier haben.

\section{Die institutionelle Gestalt}

Schließlich der Blick auf die institutionelle Gestalt der Europäischen Union. Auch hier denkt, wer die Politische Union proklamiert, in der Regel an einen großen Entwicklungssprung. Und auch hier sind es in der öffentlichen Diskussion nur wenige, immer wieder gleiche Stichworte, die den gewünschten Entwicklungssprung etwas genauer charakterisieren. Da ist zum einen die Forderung, die EU konsequent zu einer parlamentarischen Demokratie weiterzuentwickeln. Und da ist zum andern der Vorschlag, den Präsidenten der Kommission direkt wählen zu lassen - ein Vorschlag, der offenbar die präsidentielle Variante von Demokratie im Blick hat.

Kurioserweise scheint kaum wahrgenommen zu werden, dass das europäische Parlament bereits jetzt mit jenen Rechten ausgestattet ist, die für die parlamentarische Ausprägung der Demokratie konstitutiv sind. Grundlegend für das parlamentarische System ist die Regel, dass das Parlament die Regierung durch ein 
Misstrauensvotum stürzen kann. Die Rolle, die das Parlament bei der Bestellung einer Regierung spielt, ist in den Verfassungen parlamentarischer Demokratien demgegenüber durchaus unterschiedlich ausgestaltet. Das Grundgesetz sieht die Wahl des Bundeskanzlers durch den Bundestag vor, die italienische Verfassung weist ein Vertrauensvotum beider Kammern nach der Regierungsbildung aus, in Großbitannien wirkt das Parlament verfassungsrechtlich an der Regierungsbildung überhaupt nicht mit. Konstitutiv für das parlamentarische System sind diese Bestellungsregeln nicht. Stellt man das in Rechnung, ist das Europäische Parlament sogar ein besonders starkes Parlament. Denn es hat nicht nur das Recht, die Kommission zu stürzen (Art. 234 AEUV), ${ }^{7}$ sondern auch das Recht, den Kommissionspräsidenten zu wählen und die Kommission als Kollegium zu bestätigen (Art. 17 Abs.7 EUV). Das Vorschlagsrecht des Europäischen Rates fügt sich ohne weiteres in diese Deutung ein. In fast allen parlamentarischen Demokratien ist das Staatsoberhaupt mit Initiativ- und Vorschlagsrechten an der Regierungsbildung beteiligt, da und dort durchaus einflussreich, wenn vielleicht auch nicht mit dem Gewicht, mit dem der Europäische Rat bei der Auswahl des Kommissionspräsidenten tätig wird.

Wenn sich auf europäischer Ebene trotz dieser Gegebenheiten kein parlamentarisches System herausgebildet hat, so kann die Erklärung dafür nur lauten: Die Regeln sind notwendige, aber nicht hinreichende Bedingungen für die parlamentarische Regierungsweise. Eine gewisse Bedeutung kommt sicher der Tatsache zu, dass die Kommission, ihres Initiativrechtes ungeachtet, keineswegs eine Regierung ist, sondern - das jedenfalls ist der harte Kern ihrer Aufgaben - eine Behörde, die die Einhaltung des europäischen Rechts überwacht, den europäischen Binnenmarkt administriert und seine Außenbeziehungen gestaltet. Wichtiger aber ist: Die parlamentarische Regierungsweise setzt lebendigen Parteienwettbewerb voraus, ein Parteiensystem also, das den Wettbewerb trägt, und eine Agenda, an der sich Wettbewerb entwickeln kann. Angewiesen ist der für das parlamentarische System charakteristische, sich immer wieder neu auf Zeit im Parlament im Gegenüber von Mehrheit und Minderheit formierende Parteienwettbewerb seinerseits auf einen allen zugänglichen öffentlichen Raum, in dem die politische Agenda sich bildet und zur Diskussion steht. Einen solchen Raum wiederum kann sich nur eine Kommunikationsgemeinschaft schaffen.

7 Zwar verlangt der Vertrag dafür zwei Drittel der abgegebenen Stimmen und zugleich die Mehrheit der Stimmen der Mitglieder des Europäischen Parlamentes. Aber auch das Grundgesetz schreibt für das konstruktive Misstrauensvotum eine absolute Mehrheit vor. 
Alle diese Voraussetzungen sind im Großraum Europa allenfalls rudimentär gegeben. Sie lassen sich auch nicht einfach durch Willensakt schaffen. Genau das spiegelt sich wider in der Wirklichkeit eines Parlamentes, das, in scheinbarem Widerspruch zu den verfassungsrechtlichen Gegebenheiten, der Funktionslogik des parlamentarischen Systems nicht folgt. Oft wird in diesem Zusammenhang auf das fehlende Initiativrecht des Europäischen Parlamentes hingewiesen. Natürlich ist das eine Besonderheit der europäischen Verfassungskonfiguration. Aber eine Erklärung dafür, dass sich im politischen System der EU die parlamentarische Regierungsweise nicht entwickelt hat, obwohl das Parlament über die einschlägigen Rechte verfügt, ist es nicht. Faktisch ist die Gesetzesinitiative in den meisten parlamentarisch verfassten Demokratien fast ganz auf die Regierung übergegangen.

Der Ruf, die EU zu einer parlamentarischen Demokratie weiterzuentwickeln, geht also in gewisser Weise ins Leere. Die Verfassungsvoraussetzungen dafür sind bereits gegeben, die gesellschaftlich-politischen, über die man nicht einfach Beschluss fassen kann, aber eben nicht. So richtet sich das Interesse auf jenen zweiten Vorschlag der institutionellen Ausgestaltung einer politischen Union: die direkte Wahl des Kommissionspräsidenten. Man hat mit Recht darauf hingewiesen, dass dieser Vorschlag der Gewaltenteilungsstruktur, so wie sie sich im politischen System der EU tatsächlich entwickelt hat, sehr viel besser entspricht als der Vorschlag einer Parlamentarisierung der EU, der diese Struktur schlicht ignoriert. ${ }^{8}$ Das Europäische Parlament ist eben kein eine Regierung stützendes und tragendes Parlament, es ist vielmehr eine Art von Gegenmacht zu Kommission und Ministerrat. Es übt seine Gesetzgebungsrechte sehr unabhängig aus, ohne Loyalitätsbindung an die Kommission. Es ist eine Instanz der Kontrolle und braucht dazu die Unabhängigkeit von der Kommission. Man denkt an den US-Kongress, wenn man nach Parallelen in der Staatenwelt sucht, nicht an Parlamente parlamentarisch verfasster Demokratien. Und so ist es ganz schlüssig, dass der Vorschlag, den Kommissionspräsidenten direkt wählen zu lassen, gleichsam den Spuren der amerikanischen, präsidentiellen Variante der Demokratie folgt. $^{9}$

8 Maurer, A.: Der Preis der Stärke, in: Frankfurter Allgemeine Zeitung, 21.10.2013, 7, weist zutreffend darauf hin, dass die konsequente Parlamentarisierung der Europäischen Union keine Fortentwicklung der europäischen Verfassung wäre, sondern gerade umgekehrt ein Bruch mit der bisherigen Entwicklung darstellte.

9 Darauf macht Decker aufmerksam. Vgl. Decker, F.: Demokratie und Demokratisierung jenseits des Nationalstaates. Das Beispiel der Europäischen Union, in: Zeitschrift für Politikwissenschaft, 10 (2000), 585-629. 
Aber dieser Stimmigkeit ungeachtet wäre es ganz unrealistisch, im präsidentiellen Demokratietypus ein geeignetes Muster für die Fortentwicklung des politischen Systems der EU zu sehen. Auch hier gilt: Es fehlen wesentliche gesellschaftlich-politische Voraussetzungen. Und es ist eine gefährliche Illusion anzunehmen, die Regeln könnten aus sich heraus ihre eigenen systemischen Voraussetzungen schaffen. Ohne den systemischen Kontext einer lebendigen Wettbewerbsdemokratie, der ihr ihre Bedeutung gibt, kann die Direktwahl des Kommissionspräsidenten nur zu Enttäuschungen führen. Denn sie würde vom Wähler mit Erwartungen überfrachtet, die sie nicht einlösen könnte. Der amerikanische Präsident, so umstritten seine Politik auch sein mag, ist doch auf eine einzigartige Weise Repräsentant der Nation. Ein Kommissionspräsident, der die Mehrheit seiner Wähler nur über Dolmetscher ansprechen kann, könnte für die Völker Europas etwas Ähnliches niemals sein. Und wie sollte sein Wahlprogramm aussehen? Ist angesichts scharfer Interessengegensätze zwischen den Mitgliedstaaten in wichtigen Fragen überhaupt ein nicht nur auf Floskeln beschränktes Wahlprogramm denkbar, das in allen Staaten präsentiert werden könnte? Träten die Kandidaten aber mit substantiellen Programmen an, könnten sie sie einlösen? Im Gewaltenteilungssystem der EU sind die Handlungsmöglichkeiten des Kommissionspräsidenten viel begrenzter als die des amerikanischen Präsidenten in der amerikanischen Verfassungsordnung. Wirkliche Weichenstellungen in der Entwicklung der Europäischen Union, die der Legitimation durch den Wähler bedürfen, sind nicht seine Sache, sondern dem Europäischen Rat vorbehalten. Es ist zweifelhaft, ob der Wählerentscheidung, abgesehen von der Übertragung des Amtes auf eine Person, überhaupt irgendwelche bedeutsamen politischen Folgen zugerechnet werden könnten. Wie aber lässt sich dann ein solcher symbolisch hoch aufgeladener Akt rechtfertigen?

Eines gilt für beide Konzeptionen institutioneller Weiterentwicklung der EU: Sie orientieren sich an nationalstaatlichen Demokratiemodellen. Wir stoßen, heißt das, auch hier wieder auf die Vorstellung, dass es mit der Europäischen Union, zu Ende gedacht, auf ein staatliches Gebilde hinauslaufen müsse. Dem Sog des Modells Staat ist, wird über die Zukunft Europas nachgedacht, offenbar kaum zu widerstehen - das ist in der Debatte über die Politische Union auf Schritt und Tritt zu spüren. Überraschend ist das nicht. Der Staat ist nun einmal in der Neuzeit zu der - nicht nur unser Leben, sondern auch unser Denken beherrschenden - Form der politischen Organisation menschlichen Zusammenlebens schlechthin geworden. Und doch ist es auch wieder überraschend, weil der Aufbau einer europäischen Föderation in der zweiten Hälfte des 20. Jahrhunderts ja von der 
Erfahrung angestoßen und geleitet war, dass der Staat mit seinen Möglichkeiten an Grenzen stößt und dass insbesondere der traditionelle Staatenantagonismus in Europa zu katastrophalen Fehlentwicklungen geführt hat; dass es also offenbar nicht einfach nur um einen neuen Staat anderer Größenordnung gehen kann.

\section{Ist ein Staat Europa ein sinnvolles Ziel?}

Die Konturen des Programms zur Staatswerdung Europas, wie es sich hinter dem Ruf nach einer Politischen Union abzeichnet, mögen deutlicher geworden sein. Die Frage, ob der Staat Europa ein erreichbares und vernünftiges Ziel ist, bleibt noch zu stellen. Die überwiegend enthusiastische Bejahung dieser Frage in den frühen Phasen der Integrationsgeschichte ist in den vergangenen beiden Jahrzehnten einer wachsenden Skepsis gewichen. Die Zweifel daran, dass ein Staat Europa ein realistisches oder, wichtiger noch, ein erstrebenswertes Ziel sei, werden vielfältig artikuliert. ${ }^{10}$ Es genügt, in wenigen Worten an sie zu erinnern.

Die einfachste Feststellung ist zugleich die zwingendste: Eine Föderation von beinahe 30 Nationalstaaten, jeder mit seiner eigenen Sprache, seiner eigenen Geschichte, seiner besonderen kulturellen und politischen Prägung, kann nicht ein Bundesstaat im herkömmlichen Verständnis werden. Man hat, aus guten Gründen, die Erweiterung der Föderation bis an die Grenzen des Kontinents vorwärtsgetrieben, hat der Erweiterung der Union, heißt das, den Vorrang vor der Vertiefung eingeräumt. Damit sind irreversibel Weichen gestellt. Staatswerdung ist für diese Föderation in ihrer Vielgliedrigkeit und Vielgestaltigkeit keine Option mehr. Etwas anders formuliert: Die Völker Europas sind politisch - unter dem Dach Europas - primär in ihren eigenen Staaten zu Hause, sehen in diesen das Gehäuse ihrer kollektiven politischen Identität. Und wenn nicht, wie etwa in Flandern, Katalonien oder Schottland, geht es eher um kleinere politische Einheiten, nicht um größere. Die Idee einer Staatswerdung Europas geht nicht von den Völkern Europas aus und wird nicht von ihnen getragen.

In dieser Bindung an den Nationalstaat ist vermutlich nicht nur die Neigung wirksam, im Hergebrachten zu verharren, wie man kritisch meinen könnte, sondern auch so etwas wie eine wohl begründete Ahnung, dass ein kontinentaler Großstaat, ein Staat der 500 Millionen mit 20 Sprachen, wie immer er im Einzelnen verfasst sein mag, nicht ohne erhebliche Verluste an demokratischer Sub-

10 Ein charakteristisches Beispiel für die größer gewordene, sehr prinzipiell begründete Skepsis gegenüber der Idee, dass die Einigung Europas letztlich auf einen Staat hinauslaufen sollte: Lübbe, H.: Abschied vom Superstaat. Vereinigte Staaten von Europa wird es nicht geben, Berlin, 1994. 
stanz zu haben sein würde; eine Ahnung auch, dass sich in einem europäischen Bundesstaat nach aller Erfahrung eine unerträgliche Zentralisierungsdynamik entwickeln würde - bis heute ist es ja trotz aller verbalen Beschwörungen nicht gelungen, in der EU Mechanismen der verlässlichen Gewährleistung von Subsidiarität zu schaffen; eine Ahnung schließlich, dass das Einheitsrecht für ganz Europa, welches das Hauptprodukt der Europäischen Union ist, vielleicht doch nicht in allen Fällen die besseren Problemlösungen bietet. Wenn es sich so verhält, kann man die Staatswerdung Europas auch nicht wünschen.

Aber wenn die Staatswerdung nicht die Perspektive der Europäischen Föderation ist, was ist dann ihre Perspektive? Es gilt, die unendlich oft und floskelhaft wiederholte Formel vom Gebilde sui generis ernst zu nehmen. Die Europäische Union ist einzigartig, und das nicht zufällig, sondern aus guten Gründen. Sie ist kein, wie Walter Hallstein es noch wollte, „unvollendeter Bundesstaat“. Alle vertrauten Modelle politischer Organisation erweisen sich für das europäische Projekt als Prokrustes-Bett.

\section{Die Einzigartigkeit der Föderation europäischer Staaten}

Was aber heißt sui generis konkret? In der Europäischen Union ist das Zusammenwirken von Staaten auf bestimmten Politikfeldern in einer Weise dauerhaft institutionalisiert, die die Staatenwelt bis zur Gründung der Europäischen Föderation noch nicht kannte und bis heute nur in diesem einen einzigen Fall kennt. Zwei Gruppen von Akteuren stehen in diesem Kooperationssystem einander gegenüber: die Mitgliedstaaten und die von ihnen geschaffenen, dann aber in die Unabhängigkeit entlassenen supranationalen Institutionen. Dabei sind drei Modi des politischen Handelns der Union zu unterscheiden: der Modus souveränen Handelns der Mitgliedstaaten, der Modus souveränen Handelns der supranationalen Institutionen und der Modus des Zusammenwirkens von Mitgliedstaaten und supranationalen Institutionen. Souverän, aber doch eingeordnet in das institutionelle Gehäuse der Union und gebunden an die Zielvorgaben der Verträge, auch an gewisse Verfahrensvorschriften, agieren die Mitgliedstaaten in der Fortschreibung der Vertragsverfassung der EU, in den politischen Direktiven, mit denen der Europäische Rat die Entwicklung der EU lenkt, und in der gemeinsamen Außen- und Sicherheitspolitik. Souverän agieren die supranationalen Institutionen in Gestalt der Kommission bei der Überwachung, Administration und Außenvertretung des Gemeinsamen Marktes, in Gestalt des EuGH bei der verbindlichen Auslegung des europäischen Rechtes und in Gestalt der EZB und des ESM bei der Administration und Stützung der gemeinsamen Währung. Gemein- 
sam handeln die Mitgliedstaaten (durch den Rat) und die supranationalen Institutionen (in Gestalt von Parlament und Kommission) vor allem in der europäischen Gesetzgebung, wobei für dieses gemeinsame Handeln, was den Rat angeht, teils (und überwiegend) die Mehrheitsregel, teils die Regel der Einstimmigkeit gilt, die allen Staaten ein Vetorecht verleiht. Dabei hat die Mitwirkung der Staaten Schlüsselbedeutung für die Legitimation europäischen Entscheidens, während die supranationalen Einrichtungen eine primäre Verantwortung für seine Effizienz tragen. Gewiss auch für das, was man die europäische Dimension europäischen Entscheidens nennen könnte. Aber wenn man darauf hinweist, sollte man gleich hinzufügen: Unverzichtbar ist auch der Dialog unter den Mitgliedstaaten, die Suche nach Kompromissen zwischen ihnen, in denen „die europäische Dimension europäischen Entscheidens“ ausfindig gemacht wird und ausfindig gemacht werden muss.

Um die Besonderheit dieser Ordnung an einem Detail noch einmal zu veranschaulichen: Zu den Verfassungsvorschlägen, die sich mit dem Stichwort Politische Union verbinden, gehört auch der, den Ministerrat nach bundesstaatlichem Muster zu einer „normalen“ Länderkammer umzubilden. Auch hier wieder die Tendenz, sich an vertrauten Mustern der Staatlichkeit zu orientieren. Bundesstaaten haben neben dem nach allgemeinem und gleichem Wahlrecht gewählten Parlament, das das Bundesvolk repräsentiert, eine Länderkammer, in der die Gliedstaaten, in aller Regel mit dem gleichen Stimmgewicht, vertreten sind - der deutsche Bundesrat mit seiner schwachen Proportionalität ist die große Ausnahme. Dieses Schema eignet sich für die Europäische Union aber eben gerade nicht. Im Europäischen Parlament kann das Postulat des gleichen Stimmgewichts für alle Unionsbürger nur höchst unvollkommen verwirklicht werden, weil dieses Postulat, gesteht man den sehr kleinen Mitgliedstaaten eine angemessene Mindestzahl von Mandaten zu, zu einer Riesenversammlung führen würde, die nicht mehr arbeitsfähig wäre. Der Ministerrat andererseits kann, gewissermaßen kompensatorisch für die Ungleichheiten der Repräsentation im Parlament, nicht strikt nach dem Muster der Gleichheit aller Staaten, ob groß oder klein, organisiert werden. Denn die Staaten sind die wesentlichen Vermittler von Legitimität für die europäischen Entscheidungen. Das können sie aber nur sein, wenn die extrem unterschiedlichen Bevölkerungszahlen nicht völlig ignoriert werden. Die Abstimmungsregel der doppelten Mehrheit (Art. 238 AEUV) ist eine intelligente Lösung dieses Problems und nicht nur eine Abweichung von der Normalität bundesstaatlicher Verfahrensregeln, die allenfalls vorübergehend hinnehmbar ist. Es gilt, um es noch einmal zu sagen, das sui generis ernst zu nehmen. 
Diese überaus komplexe, über Jahrzehnte immer wieder neu ausgehandelte, zugleich aber auch in ihrer Entwicklung einer eigenen inneren Dynamik folgende Verfassungsarchitektur lässt sich in gewissem Sinne geradezu als ein Musterfall jenes Typus von politischer Ordnung verstehen, den Edmund Burke, die englische Verfassung den Verfassungsentwürfen der Französischen Revolution gegenüberstellend, so idealisiert hat: ${ }^{11}$ nicht am Reißbrett entworfen, sondern gewachsen; ein Werk von Generationen, nicht eines einzigen Architekten; die Quintessenz langer Erfahrung, nicht der Ratio eines Augenblicks entsprungen. Anders als auf eben diesem Weg hätte sich das Unerhörte - fast 30 ursprünglich souveräne Staaten haben sich inzwischen einer gemeinsamen Rechtsordnung unterworfen - wohl kaum ereignen können.

Dass Burke die europäische Vertragsverfassung, so wie sie sich langsam, Schritt für Schritt herausgeformt hat, wohl für ein Produkt historischer Vernunft gehalten hätte, hilft ihr wenig. Es wird fast nur unfreundlich über sie geredet. Den einen ist die Integrationsentwicklung in vielen Hinsichten schon zu weit fortgeschritten, den anderen, den Enthusiasten des „immer enger“ - und um deren Argumente geht es hier - ist die Stellung der Mitgliedstaaten im europäischen Verfassungsgefüge noch immer viel zu stark. Das hat speziell im deutschen Fall mit - historisch natürlich gut erklärbaren - tiefen Ressentiments gegen den Nationalstaat als solchem zu tun, Ressentiments, denen es ein Ärgernis ist, dass Nationalstaat und Demokratie viel miteinander zu tun haben, auch heute noch. ${ }^{12}$ Aber es hat auch und, wenn wir vom Sonderfall Deutschland absehen, vermutlich zuerst mit zwei gravierenden Defiziten der EU in ihrer gegenwärtigen Verfassungsgestalt zu tun - jedenfalls ist das die Sicht der meisten Plädoyers für das Integrationsziel Politische Union. Es fehle der EU an Handlungsfähigkeit, ist das eine Monitum. Das, was man das europäische Gemeininteresse nennen könnte, sei in den Prozessen europäischer politischer Willensbildung nicht stark genug, das andere.

In der Tat - hier sind zwei wirkliche Schwächen der europäischen Föderation angesprochen. Aber doch nur sehr pauschal. Die Feststellung, der EU fehle es an Handlungsfähigkeit, wird erst dann zu einer weiterführenden Diagnose, wenn die Frage beantwortet wird, welche europäischen Kapazitäten denn um welcher Problemlagen willen genau gebraucht werden. Europäische Lösungen sind nicht

11 Burke, E.: Betrachtungen über die Französische Revolution, Zürich, 1986.

12 Ein Beispiel: Harpprecht, K.: Der Nationalstaat war gestern, in: Frankfurter Allgemeine Zeitung, 15.08.2013, 6. 
schon deshalb generell die besseren, weil es europäische Lösungen sind. Kann man etwa im Blick auf die Sicherheitspolitik oder die Energiepolitik mit guten Gründen argumentieren, dass eine Stärkung der EU wünschenswert sei, so gilt das für eine weitere Verdichtung des vereinheitlichenden europäischen Binnenmarktrechts keineswegs. Genau in diese Richtung aber drängt die Entwicklungsdynamik der EU, wohl auch, weil die Mitgliedstaaten andere, sinnvollere Entwicklungen blockieren. Im Übrigen wird mit jeder weiteren Ermächtigung der EU die Notwendigkeit, dem Subsidiaritätsprinzip endlich verlässliche Wirkung zu verschaffen, dringlicher. Bis heute ist dieses Problem ungelöst.

Was die Wirkmächtigkeit des europäischen Gemeininteresses angeht, so steht außer Zweifel: Mit der Aufgabe, dort, wo es darauf ankommt, ein europäisches Gemeininteresse zu definieren und durchzusetzen, ist die EU noch nicht weit gekommen. Aber die Schlussfolgerung, also gelte es, die supranationalen Verfassungsorgane der EU zu stärken, ist zu einfach. Diese Organe tendieren alle dazu, europäisches Gemeinwohl mit dem Gewinn zusätzlicher Kompetenzen für sich gleichzusetzen. Eben diese Tendenz macht übrigens auch das oft geforderte Initiativrecht des Europäischen Parlamentes so problematisch. Zudem ist daran zu erinnern: Für das demokratische Verständnis von Gemeinwohl ist konstitutiv, dass unterschiedliche Vorstellungen davon, was konkret gemeinwohldienlich sei, miteinander in einem durch die Wählerschaft auf Zeit zu entscheidenden Wettbewerb stehen. In einen solchen politischen Wettbewerb ist von den supranationalen Verfassungsorganen der EU allenfalls das Parlament eingebunden. Und auch das nicht wirklich, weil, wie die Dinge derzeit stehen, in den europäischen Parlamentswahlen dem Wähler keine konkurrierenden politischen Konzeptionen europäischen Gemeinwohls präsentiert werden. Schließlich ist auch darauf noch einmal hinzuweisen, dass der Versuch der supranationalen politischen Akteure, das europäische Gemeininteresse im Widerspruch zu als lebenswichtig wahrgenommenen Interessen einzelner Mitgliedstaaten zu bestimmen, das europäische Projekt nicht weiterbrächte. Er würde es zersprengen.

Ist das, mag der Leser am Ende fragen, ein Plädoyer dafür, sich mit dem Status quo zu bescheiden? Es ist zumindest ein Plädoyer gegen die Unterschätzung des Erreichten. Die europäische Föderation, verlässlicher Kooperationsverbund und Rechtsgemeinschaft von Staaten, die ihre Souveränität partiell in den Verbund eingebracht haben, sucht weltweit ihresgleichen. Das kann nicht oft genug gesagt werden. Man wird dem historischen Rang dieser Entwicklung in keiner Weise gerecht, wenn man die EU, so wie sie ist, immer nur als etwas Unfertiges, Unvollkommenes, Verbesserungsbedürftiges wahrnimmt. Natürlich wird sie sich 
weiterentwickeln. Aber es ist unwahrscheinlich, dass in einer Gemeinschaft von demnächst dreißig oder mehr Staaten, Staaten, die - von Finnland bis Portugal, von Bulgarien bis Irland - nicht sehr viel miteinander gemein haben, noch große Integrationssprünge möglich sind. Die weitere Entwicklung dieser komplexen Großgemeinschaft wird sich in starker Pfadabhängigkeit, langsam und schrittweise vollziehen. Größere Bewegungsspielräume werden sich nur da ergeben, wo von der Möglichkeit der „verstärkten Zusammenarbeit“ Gebrauch gemacht wird (Art. 20 EUV, Art. 326ff. AEUV). Wenn man überhaupt Bewegung will, wird also die Toleranz für Binnendifferenzierung in der EU größer werden müssen. Die Verträge sind freilich nicht darauf angelegt, dazu zu ermutigen. Die Bedingungen sind eher restriktiv formuliert, beginnend mit der Vorgabe, dass sich mindestens neun Mitgliedstaaten zu einer verstärkten Zusammenarbeit zusammenfinden müssen.

Ganz unabhängig von der institutionellen Entwicklung wird sich für jedes Vorhaben, den Integrationsprozess über das erreichte Niveau hinaus weiterzutreiben, die Frage stellen, wie der Sinn oder, handfester, der Nutzen des europäischen Projektes erfahrbar gemacht werden kann. Das ist, so scheint es, im Fortgang der europäischen Einigung nicht leichter, sondern schwieriger geworden. Dass der Friede in Europa gefährdet sei, wenn der Integrationsprozess nicht ständig fortschreite, glaubt, zu recht, kaum noch jemand. Es ist unklug, Europa mit einer solchen Rhetorik vorwärts bringen zu wollen. Hilfreich könnte es sein, wenn es gelänge, das europäische Projekt immer wieder in einzelne konkrete Projekte von überschaubaren Dimensionen zu übersetzen, deren Nutzen tatsächlich evident ist. Der Versuch, die EU zur Vorkämpferin einer globalen Klimapolitik zu machen, ist ein - sehr ambitioniertes - Beispiel, ein Beispiel freilich auch, das die Schwierigkeiten binneneuropäischer Konsensbildung ebenso deutlich vor Augen führt wie die Begrenztheit des europäischen Vermögens, Weltentwicklungen zu beeinflussen. Dennoch: Es könnte sein, dass es Europa gut täte, wenn es sich viel nachdrücklicher als in der Vergangenheit der Frage stellte, worin denn der Nutzen des europäischen Projektes für die Welt liege. Dass es als solches Vorbildcharakter für andere Regionen der Welt gewinnen könnte, ist nicht ausgeschlossen, aber einstweilen nicht sehr wahrscheinlich; umso unwahrscheinlicher übrigens, je mehr es als auf Staatswerdung angelegt begriffen wird. 